\title{
Improving Drug Prescription in Elderly Diabetic Patients
}

\author{
Francesc Formiga Pérez \\ Geriatric Unit, Internal Medicine Service, Hospital Universitari de Bellvitge, Barcelona, Spain
}

Conflicts of Interests: The authors declare that they have no conflicts of interest in this research.

Acknowledgements: This abstract has been presented as a lecture at $2^{\text {nd }}$ International Seminar REPOSI: Targeting the burden of polypharmacy in the elderly.

This article is licensed under a Commons Attribution Non-Commercial 4.0 License

Nowadays, there is no doubt that there is a good epidemic of ageing with the inversion of the population pyramid. There is no doubt either about the current epidemic of obesity and bad nutritional habits that have resulted in an increase of diabetic patients in the world. And the union of these epidemics has led to an increase of diabetes (DM) in the elderly.

The spectrum of comorbidities in patients with diabetes is very broad, and usually comorbidity is associated with polypharmacy. In diabetic patient polypharmacy is often unavoidable, since multiple drug therapy has become the standard for most of its common comorbidities. The potential risk of interactions caused by polypharmacy is likely to continue rising as more therapeutic options will become available.

To improve drugs prescription in elderly DM patients (Table 1) it is important to make a global evaluation and to follow the recommendations of scientific societies, always bear in mind the aim to avoid hypoglycemia. We must also know all the possible drugs very well. This applies not only to diabetes drugs, but also to medications used to control other common associations, such as cardiovascular risk factors.

It seems clear that in addition to individual strategies, global strategies are also fundamental to improve the treatment for diabetes in older people. The role of different regulatory and pharmacovigilance agencies will be essential as they will keep us informed about alerts and warnings. Not only doctors and nurses should be involved but also a pharmaceutical consultant as part of multidisciplinary teams.

Finally, we need remember the importance to focus on each particular patient and the importance to involve the patient and the family throughout the process.

\section{SET SUITABLE THERAPEUTIC TARGETS AVOID HYPOGLYCEMIAS DRUG REVIEW: DM AND GLOBAL IMPROVEMENT STRATEGIES: COMMUNITY, HOSPITAL, NURSING HOME}

Table 1: Recommendations for improving drugs prescription in diabetic patients. 